This is a pre-copyedited, author-produced version of an article accepted for publication in European Heart Journal - Quality of Care and Clinical Outcomes following peer review. The version of record Wilkinson, Chris, Weston, Clive, Timmis, Adam, Quinn, Tom, Keys, Alan and Gale, Chris P (2020) Cohort profile : the Myocardial Ischaemia National Audit Project (MINAP). European Heart Journal - Quality of Care and Clinical Outcomes, 6(1), pp. 19-22. is available online at: https://academic.oup.com/ehjqcco/ article/6/1/19/5567511 


\section{Cohort profile: The Myocardial Ischaemia National Audit Project (MINAP)}

Chris Wilkinson ${ }^{1}$, Clive Weston², Adam Timmis ${ }^{3}$, Tom Quinn ${ }^{4}$, Alan Keys ${ }^{5}$, Chris P Gale 1,6

${ }^{1}$ Leeds Institute of Cardiovascular and Metabolic Medicine, University of Leeds, Leeds, UK

${ }^{2}$ National Institute for Cardiovascular Outcomes Research, Barts Health NHS Trust, London, UK

${ }^{3}$ NIHR Cardiovascular Biomedical Research Unit, Barts Heart Centre London, London, UK

${ }^{4}$ Kingston University and St. George's, University of London, London, UK

${ }^{5}$ Patient representative on MINAP Domain Expert Group, National Institute for

Cardiovascular Outcomes Research, Barts Health London, UK

${ }^{6}$ Leeds Institute for Data Analytics, University of Leeds, Leeds, UK

Correspondence:

Professor Chris P Gale, Leeds Institute of Cardiovascular and Metabolic Medicine, University of Leeds, Leeds LS2 9JT, UK; c.p.gale@leeds.ac.uk @cpgale3 


\section{Abstract}

\section{Aims}

The Myocardial Ischaemia National Audit Project (MINAP) collects data from admissions in England, Wales and Northern Ireland with type 1 myocardial infarction. The project aims to improve clinical care through the audit process and to provide powerful high-resolution data for research.

\section{Methods and Results}

MINAP collects data spanning 130 data fields covering the course of patient care, from the moment the patient calls for professional help through to hospital discharge and rehabilitation.

Data are entered by clinicians and clerical staff within hospitals, and pseudonymised records are uploaded centrally to the National Institute for Cardiovascular Outcomes Research (NICOR), hosted by Barts Health NHS Trust, London, UK. 206 hospitals submit over 92,000 new cases to MINAP annually. Approximately 1.5 million patient records are currently held in the database.

Patient demographics, medical history, clinical assessment, investigations, treatments, drug therapy prior to admission, during hospital stay and at discharge are collected. Data completeness of three key data fields (age, admission blood pressure, and heart rate) is over $91 \%$. Vital status following hospital discharge is obtained via linkage to data from the United Kingdom Office for National Statistics. 
An annual report is compiled using these data, with individual hospital summary data included. Datasets are available to researchers by application to NICOR.

\section{Conclusion}

MINAP is the largest single-healthcare-system heart attack registry, and includes data from hospitalisations with type 1 myocardial infarction in England, Wales and Northern Ireland. It includes high-resolution data across the patient pathway, and is a powerful tool for quality improvement and research. 


\section{Introduction}

There were more than 11 million new cases of cardiovascular disease within European Society of Cardiology (ESC) member countries in 2015; a prevalence of over 83 million. ${ }^{1}$

Cardiovascular disease registries have been established to collect observational data about such patients. Often, databases were devised for clinical audit - whereby the performance of participating units or clinicians could be compared against explicit agreed standards of care. This served a quality assurance function. However, quality improvement initiatives are also facilitated, through what we have previously described as a 'Janus-like' capability ${ }^{2}$ - the use of registries to look both backwards, in order to identify variations or deficiencies in important elements of care, and forwards, to describe the effects of targeted interventions. Data collected in registries also have an important role in quantifying disease burden to assist service planning, the implementation of new therapies, and the planning of research. ${ }^{2}$ Registries have been used in the development of prognostic models, ${ }^{2}$ as a source of observational data for hypothesis formulation, and, more recently, as a relatively inexpensive method of performing randomised controlled trials - offering opportunities for recruitment, randomisation and follow-up, and providing valuable real-world context. ${ }^{3}$

The Myocardial Ischaemia National Audit Project (MINAP) is one of six domains of cardiovascular care that, together, comprise the National Cardiac Audit Programme (NCAP) within the National Institute for Cardiovascular Outcomes Research (NICOR). MINAP receives data for hospital admissions in England, Wales and Northern Ireland with type 1 myocardial infarction (T1 MI). ${ }^{4}$ The project aims to 
examine and improve clinical care, and so outcomes, of patients with acute coronary syndromes through the audit process, and to provide powerful high-resolution data for research..$^{5-12}$ It is the largest single-healthcare-system heart attack registry in the world, originally established to support a national framework for coronary heart disease, ${ }^{13,14}$ and has been collecting data since 2000 . A similar example is the Swedish Web-system for Enhancement and Development of Evidence-based care in Heart disease Evaluated According to Recommended Therapies (SWEDEHEART), which was launched in December 2009 following the merger of three existing registries. ${ }^{6,15}$

\section{Aim and Objectives}

The primary aim of MINAP is to examine and improve service delivery for, and outcomes of, patients admitted to hospital with an acute coronary syndrome. This requires the collection, cleaning, and analysis of relevant data. The results of these analyses are made available to participating hospitals, ambulance services and regional cardiac networks in a format that allows comparisons with others and with a national average, as well as benchmarking of performance against national standards or targets. This promotes and facilitates quality improvement initiatives. An annual report is released to the public. Additionally, the dataset is a resource for research which has evaluated the performance of hospitals according to the ESC Acute Cardiovascular Care Association quality indicators, ${ }^{9}$ compared care provision between men and women, ${ }^{10}$ and quantified temporal trends and geographical variations in $\mathrm{T} 1 \mathrm{Ml}$ clinical care and outcomes. ${ }^{11,12}$ 


\section{Quality of care interventions}

\section{Setting}

Presently, MINAP holds over 1.5 million records. In 2017/18 102,056 new records were submitted, of which 92,233 were confirmed T1 MI. ${ }^{16,17}$ Data are submitted by 13 ambulance services and 206 hospitals in England, Wales and Northern Ireland, figure $1 .{ }^{16}$ The costs of local data entry, including staff time for data collection and electronic data entry, are borne by the participating hospitals. Central funding for the audit activities of MINAP, including its data storage, cleaning, analyses and reporting, originate from National Health Service (NHS) England and GIG Cymru/NHS Wales, and are channelled to NICOR through a commissioned NCAP by the Health Quality Improvement Partnership (HQIP). ${ }^{18}$ Researchers pay for the costs of data extracts.

\section{Population and consent}

Participating hospitals are required to enter data for all patients admitted with $\mathrm{T} 1 \mathrm{Ml}$. Data have been continuously submitted from NHS hospitals to MINAP since October $2000 .{ }^{19}$ NICOR conforms to legislation within the Data Protection Act for the collection and use of patient-identifiable data. MINAP has approval to hold patient identifiable information without patient consent under section 251 of the NHS Act 2006.

\section{Patient identification}

Each patients' unique NHS number is pseudonymised and stored within the database. Other patient identifiers, such as local hospital number, date of birth and residential postcode, are encrypted before transmission to the central database. 


\section{Start points}

MINAP includes patients with T1 Ml. ${ }^{4}$ This includes myocardial infarction due to plaque erosion or rupture, fissure or dissection. Whilst it is possible to upload information about patients with a final diagnosis of unstable angina, the majority of cases submitted to MINAP have evidence of myocardial injury, either with or without ST-segment elevation (STEMI and NSTEMI).

\section{Baseline and follow-up data}

Data are collected within 130 fields that cover the entire patient pathway from the time the patient calls for professional help to the point of discharge, figure 2. Patient demographics, medical history and clinical assessment, investigations, treatments, drug therapy prior to admission, during hospital stay and at discharge are all included. ${ }^{20}$ The various data fields are reviewed to ensure that they remain up-todate and reflect contemporary practice. Redundant fields are archived, and continuity of data are maintained where possible..$^{21}$

\section{Data capture and storage}

Data are collected at each hospital site by clinicians and clinical audit staff. Ambulance data are abstracted from documentation completed by paramedic staff and entered onto MINAP by hospital personnel. Historically, cases were either uploaded on a case-by-case basis via a 'web portal', or entered into a PC-based package (Lotus Notes) - manually, or automatically in batches via third party software that acts in local clinical information systems. ${ }^{16}$ However, MINAP has recently adopted the Swedish IT platform, QReg5, for data entry and curation that will enable more sophisticated and timely management of the data for quality 
improvement and research. A data dictionary is available, containing explanatory detail for each field. Data are securely uploaded to NICOR, where they are stored and managed.

\section{Data quality}

There is evidence that reporting of $\mathrm{T} 1 \mathrm{MI}$ within MINAP may be incomplete. ${ }^{8}$ Yet, in the most recent MINAP annual report the overall case ascertainment rate, comparing MINAP submissions with official National Health Service (NHS) administrative hospital coding data, was $101.7 \% .{ }^{17}$ There is variation in case ascertainment between hospitals, in that 11 units (5.3\%) had rates above 150\% (suggesting greater MINAP submissions than coded discharges) and 11 (5.3\%) units had rates below $50 \%$ (suggesting suboptimal case ascertainment). The authors of the most recent report point out that this reflects a comparison based upon a fairly restrictive, albeit recommended, set of International Classification of Diseases (ICD) codes. ${ }^{17}$ Were some participating hospitals to be using a broader range of ICD codes to identify cases of AMI, as seems likely, a lower case ascertainment rate would be reported.

Data submissions should meet the minimum standard for data completeness. This is defined as at least $95 \%$ completion of 48 specified data fields for STEMI, and 43 fields for NSTEMI. ${ }^{22}$ The MINAP data application used by hospital staff contains error-checking mechanisms designed to minimise common errors. In 2015/16, data completeness of three key data fields (age, admission blood pressure, and heart rate) was $91.5 \% .{ }^{23}$ Completeness of a variety of other data fields is monitored and made available to participating centres. 


\section{Endpoints and linkages to other data}

All-cause mortality is available, as data are linked to the United Kingdom Office for National Statistics using individual patient NHS numbers to obtain regular mortality updates. Linkage between other databases held within NICOR is possible. Data are available for research and audit by application to NICOR, https://www.ucl.ac.uk/nicor/access. Applications are reviewed by a NICOR Research Group and subsequently, if approved, by the Data Access Request Group at the Healthcare Quality Improvement Partnership.

\section{Conclusion}

MINAP is the largest single-healthcare-system heart attack registry, and includes data from hospitalisations with MI in England, Wales and Northern Ireland. It holds high-resolution data across the patient pathway, with robust linked mortality data from the United Kingdom Office for National Statistics. It is a powerful tool for quality improvement within the NHS and in producing high impact research. 


\section{Figures}

Figure 1: Geoplot of hospitals and the number of patients with type 1 myocardial infarction submitted to MINAP (England and Wales

only)

Number of MINAP submissions for hospitalised

type 1 acute myocardial infarction in 2016/17

○ $1-700$ cases

701- 1400 cases

$1401-2037$ cases

Ambulance Trust Boundary

(England only)
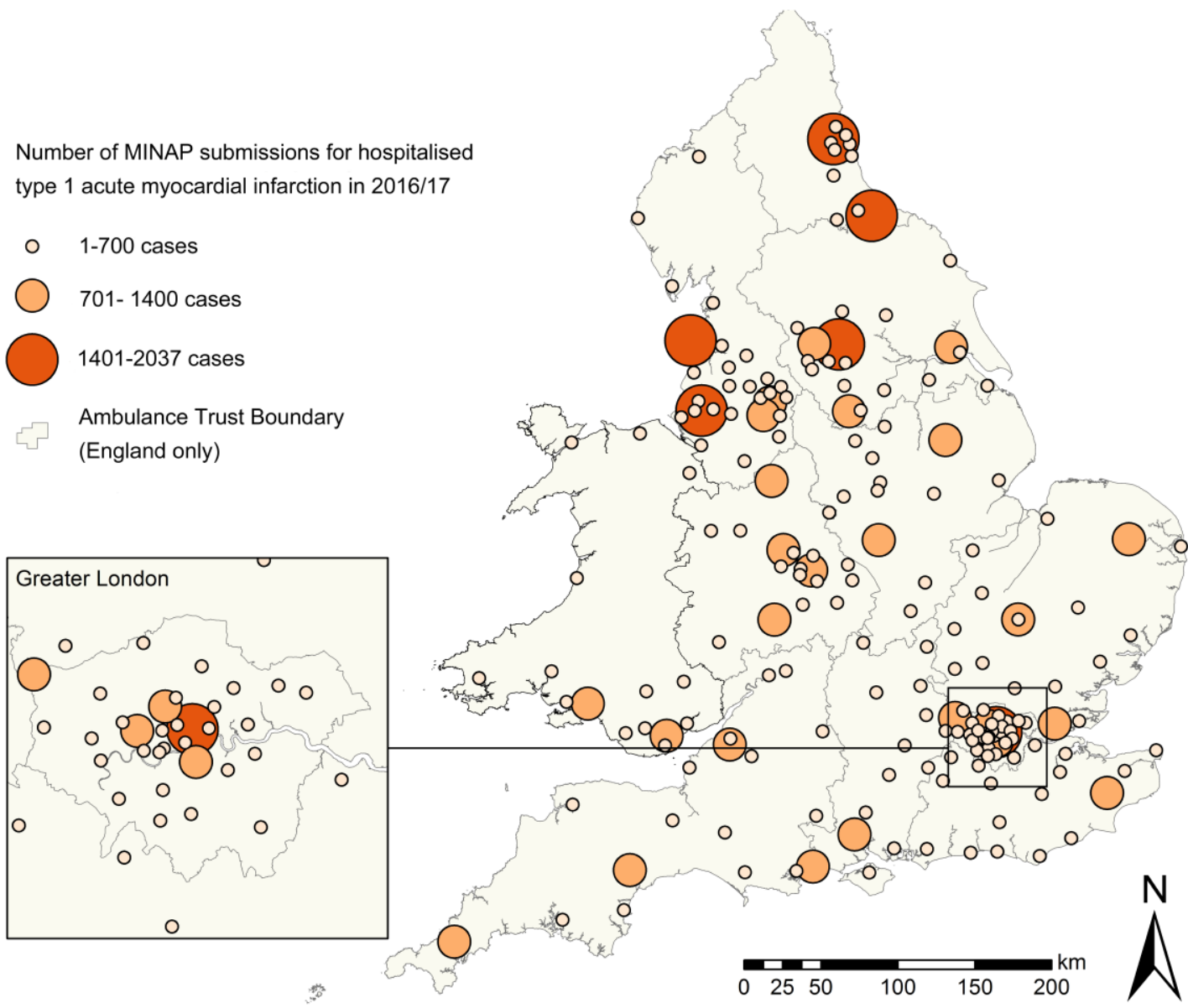


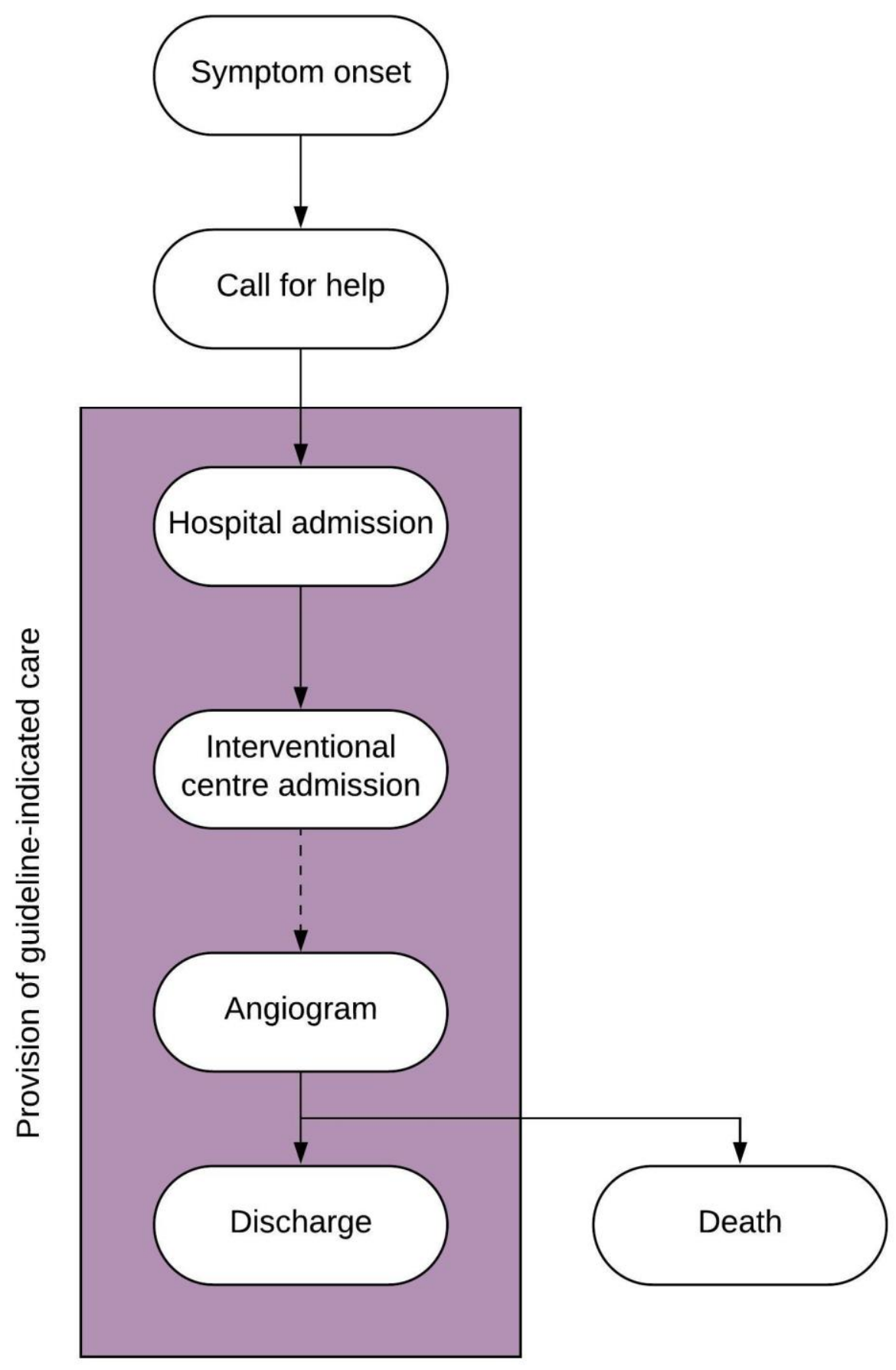

Figure 2: Data are collected at each stage of the patient journey, from symptom onset to discharge. 


\section{Acknowledgements}

Many thanks to $\mathrm{Dr}$ Thomas Burgoine for creating the GeoPlot in figure 1.

We gratefully acknowledge the contributions of all hospitals and healthcare professionals who participate in the MINAP registry, and the valuable role of the MINAP team in NICOR who manage and store the database.

\section{Funding}

C Wilkinson is funded by York Teaching Hospital NHS Foundation Trust and the Hull-York Medical School

\section{Conflicts of interest}

C Weston is Clinical Lead for MINAP. A Timmis, T Quinn, A Keys and CP Gale are members of the MINAP Domain Expert Group. CP Gale is a member of the NICOR Research Group.

\section{References}

1. Timmis A, Townsend N, Gale C, Grobbee R, Maniadakis N, Flather M et al. European Society of Cardiology: Cardiovascular Disease Statistics 2017. European Heart Journal 2018;39(7):508-579.

2. Weston C. Recycling existing data: a greener future for clinical registries. European Heart Journal - Quality of Care and Clinical Outcomes 2018;4(3):145-147.

3. Fröbert $\mathrm{O}$, Lagerqvist $\mathrm{B}$, Olivecrona GK, Omerovic $\mathrm{E}$, Gudnason $\mathrm{T}$, Maeng $\mathrm{M}$ et al. Thrombus Aspiration during ST-Segment Elevation Myocardial Infarction. New England Journal of Medicine 2013;369(17):1587-1597.

4. Thygesen K, Alpert JS, Jaffe AS, Chaitman BR, Bax JJ, Morrow DA et al. Fourth universal definition of myocardial infarction (2018). European Heart Journal 2018:ehy462ehy462. 
5. Hall M, Dondo TB, Yan AT, Mamas MA, Timmis AD, Deanfield JE et al. Multimorbidity and survival for patients with acute myocardial infarction in England and Wales: Latent class analysis of a nationwide population-based cohort. PLOS Medicine 2018;15(3):e1002501.

6. Chung S-C, Gedeborg R, Nicholas O, James S, Jeppsson A, Wolfe C et al. Acute myocardial infarction: a comparison of short-term survival in national outcome registries in Sweden and the UK. The Lancet 2014;383(9925):1305-1312.

7. Hall M, Dondo TB, Yan AT, Goodman SG, Bueno H, Chew DP et al. Association of clinical factors and therapeutic strategies with improvements in survival following non-stelevation myocardial infarction, 2003-2013. JAMA 2016;316(10):1073-1082.

8. Herrett E, Shah AD, Boggon R, Denaxas S, Smeeth L, van Staa T et al. Completeness and diagnostic validity of recording acute myocardial infarction events in primary care, hospital care, disease registry, and national mortality records: cohort study. BMJ : British Medical Journal 2013;346.

9. Bebb O, Hall M, Fox KAA, Dondo TB, Timmis A, Bueno $\mathrm{H}$ et al. Performance of hospitals according to the ESC ACCA quality indicators and 30-day mortality for acute myocardial infarction: national cohort study using the United Kingdom Myocardial Ischaemia National Audit Project (MINAP) register. European Heart Journal 2017;38(13):974-982.

10. Wilkinson C, Bebb O, Dondo TB, Munyombwe T, Casadei B, Clarke S et al. Sex differences in quality indicator attainment for myocardial infarction: a nationwide cohort study. Heart 2018.

11. Gale CP, Allan V, Cattle BA, Hall AS, West RM, Timmis A et al. Trends in hospital treatments, including revascularisation, following acute myocardial infarction, 2003-2010: a multilevel and relative survival analysis for the National Institute for Cardiovascular Outcomes Research (NICOR). Heart 2014;100(7):582-589.

12. Dondo TB, Hall M, Timmis AD, Yan AT, Batin PD, Oliver G, et al. Geographic variation in the treatment of non-ST-segment myocardial infarction in the English National Health Service: a cohort study. BMJ Open 2016;6(7):e011600.

13. Birkhead JS, Norris RM, Quinn T, Pearson $M$, on behalf of the National Service Framework for Coronary Heart Disease Steering Group. Acute myocardial infarction: a core dataset for monitoring standards of care. In: Royal College of Physicians, London; 1999.

14. National service framework for coronary heart disease. In. London: Department of Health; 2000.

15. Jernberg T, Attebring MF, Hambraeus K, Ivert T, James S, Jeppsson A et al. The Swedish Web-system for Enhancement and Development of Evidence-based care in Heart disease Evaluated According to Recommended Therapies (SWEDEHEART). Heart 2010;96(20):1617-1621.

16. National Institute for Cardiovascular Outcomes Research (NICOR). Data collection in MINAP. http://www.ucl.ac.uk/nicor/audits/minap/accordion-about/Data-collection.

17. National Institute for Cardiovascular Outcomes Research (NICOR). Myocardial Ischaemia National Audit Project 2017/18 summary report. 2019.

18. National Institute for Cardiovascular Outcomes Research (NICOR). National Cardiac Audit Programme. https://www.nicor.org.uk/national-cardiac-audit-programme/.

19. Birkhead J. Where are we today? Early results from MINAP, the National Audit of Myocardial Infarction Project. Heart 2003;89 Suppl 2:ii13-5; discussion ii35-7.

20. National Institute for Cardiovascular Outcomes Research (NICOR). MINAP datasets. http://www.ucl.ac.uk/nicor/audits/minap/datasets. 
21. Herrett E, Smeeth L, Walker L, Weston C. The Myocardial Ischaemia National Audit Project (MINAP). Heart 2010;96(16):1264-1267.

22. National Institute for Cardiovascular Outcomes Research (NICOR). MINAP minumum data standard user guidance.

https://www.ucl.ac.uk/nicor/audits/minap/documents/datasets/minap-minimum-datastandard-user-guidance-v2.0.

23. Healthcare Quality Improvement Partnership. Myocardial Ischaemia National Audit Project Annual Report April 2015 - March 2016. 2017. 
Number of MINAP submissions for hospitalised type 1 acute myocardial infarction in 2016/17

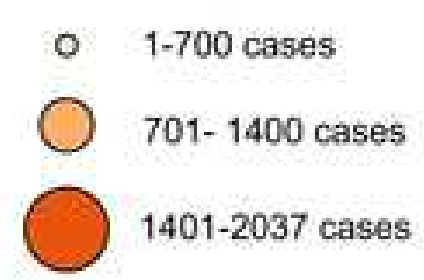

Ambulance Trust Boundary (England only)
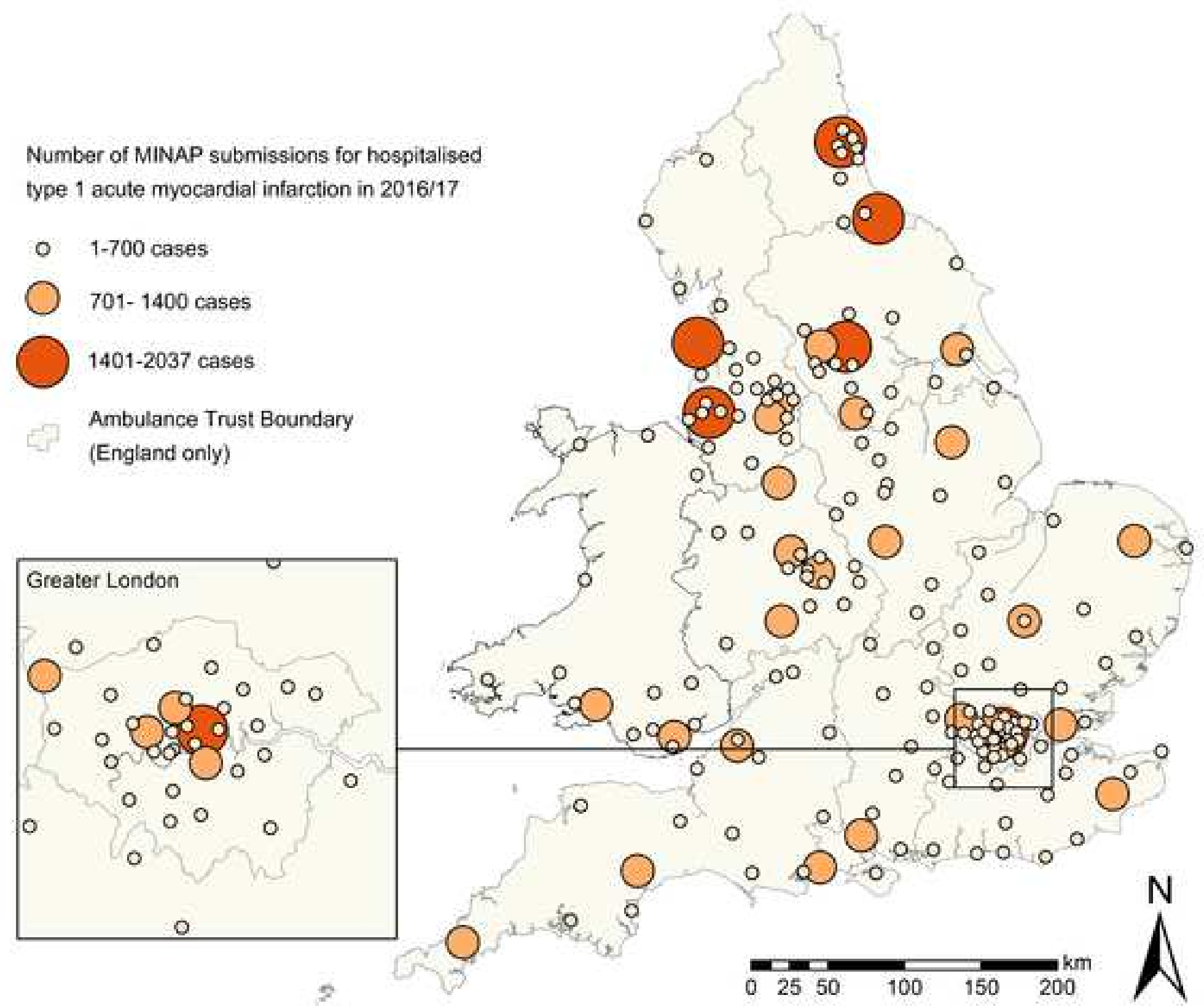


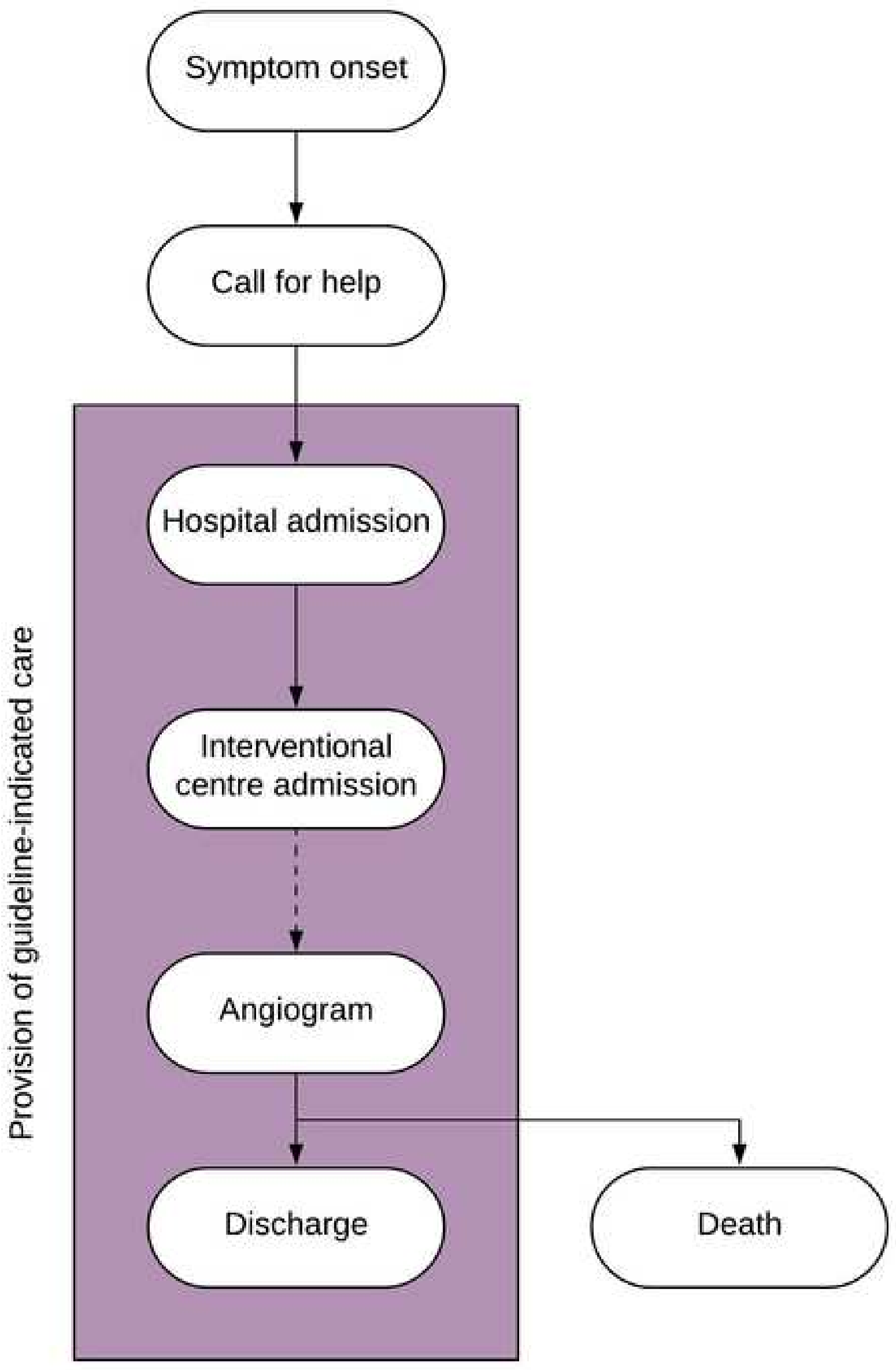

